\title{
EOPs: Predictores de recurrencias de un trastorno depresivo mayor
}

Predictors of major depressive disorder recurrences

Nicolás Lescano*

\section{Resumen}

Acude la consulta un paciente de 40 años con antecedentes de Trastorno Depresivo Mayor (TDM) diagnosticado y tratado satisfactoriamente un año atrás. Esto motiva la pregunta: En pacientes con antecedentes de TDM, ¿qué predictores pueden evaluarse para estimar el riesgo de recurrencias?. Se presenta una introducción a la problemática, la estrategia de búsqueda utilizada y el resumen de la evidencia.

Se concluye que los predictores más relevantes de recurrencias de TDM serían el número de episodios depresivos previos (OR 1,64; IC95\% 1,17 a 2,29), la presencia de síntomas subclínicos residuales (OR 3,65; IC95\% 1,17 a 2,29), los antecedentes de abuso de sustancias (OR 7,48; IC95\% 1,42 a 39,43), y/o haber sido discriminado (OR 2,92; IC95\% 1,05 a 8,11) o abusado durante la infancia (OR 1,58; IC95\% 1,05 a 2,38).

\section{Abstract}

A 40 year old patient with a history of Major Depressive Disorder (MDD) successfully diagnosed and treated one year ago attends to medical consultation. This vignette raises the question: "In patients with a history of MDD, which predictors can be assessed in order to calculate the risk of recurrences?. It is presented an introduction to the problematic, the implemented search strategy and the summary of the evidence.

It is concluded that the most relevant predictors of MDD recurrences are the number of previous depressive episodes (OR $1.64 ; \mathrm{Cl} 1,17-2,29)$, the presence of subclinical residual symptoms (OR 3.65; $\mathrm{Cl} 1.17-2.29$ ) the history of drug abuse (OR 7.48; $\mathrm{Cl} 1.42-39.43)$, and/or having experienced discrimination (OR 2.92; Cl 1.05-8.11) and abuse during childhood (OR 1.58; Cl 1.05-2.38).

Palabras clave: depresión, recurrencia, factores de riesgo. Key words: depressive disorder, recurrence, risk factors.

Lescano N. Predictores de recurrencias de un trastorno depresivo mayor. Evid Act Práct Ambul. Jul-Set 2012;15(3):118-119.

\section{Escenario clínico}

Un médico de atención primaria recibe en su consultorio a un paciente de 40 años de edad con antecedentes de Trastorno Depresivo Mayor (TDM). El paciente fue diagnosticado un año atrás y recibió tratamiento satisfactorio, alcanzando una recuperación completa. Actualmente no recibe ningún tratamiento psicofarmacológico ni psicoterapéutico. El médico sabe que la Depresión Mayor (DM) puede ser recurrente y se cuestiona acerca de qué factores o características de su paciente puede indagar para estimar su riesgo de tener una recurrencia.

\section{Pregunta}

En pacientes con antecedentes de TDM, ¿qué predictores pueden evaluarse para estimar el riesgo de recurrencias?

\section{Estrategia de búsqueda}

Se realizó una búsqueda en Trip Database utilizando las palabras clave "major depression AND (recurrence OR relapse) AND predictors" que arrojó 173 resultados. Entre los de las categorías Evidence Based Synopsis (8 resultados), Systematic Reviews (7) y Guidelines (47) ninguno respondía satisfactoriamente a la pregunta formulada.

No se encontró ningún resultado en las categorías Clinical Q\&A ni Core primary research.

Por lo tanto fueron analizados los resúmenes de los artículos encontrados en la categoría Extended primary research (46), lo que condujo a la preselección de seis artículos como potencialmente adecuados para responder la pregunta. De estos últimos, resumiremos los dos que brindaron la información de mayor relevancia.

\section{Introducción al problema}

Los trastornos depresivos representan la principal causa mundial de discapacidad y contribuyen en cuarto lugar con la carga mundial de enfermedad, estimándose que ocuparán el segundo lugar en $2020^{1}$. El TDM presenta una prevalencia de entre el $10 \%$ y el $25 \%$ en las mujeres y entre el $5 \%$ y el $12 \%$ en los hombres de la población general².

Es importante destacar que por tratarse de un trastorno con una naturaleza crónica y recurrente, sumada a su elevada prevalencia y morbimortalidad, se justifica una evaluación cuidadosa del paciente y un tratamiento adecuado de sus eventuales recurrencias. Se plantean diferentes tratamientos con fases de continuación y mantenimiento tras la remisión del cuadro, con el fin de evitar recaídas o recurrencias.

Los términos recaída (en inglés: relapse) y recurrencia (recurrence) han sido confundidos durante cierto tiempo, pero se han estandarizado de la siguiente manera: se denomina recaída al retorno de síntomas durante el período de remisión anterior a la recuperación (durante las ocho primeras semanas de iniciado el tratamiento) y recurrencia a la aparición de un nuevo episodio depresivo después del período de recuperación (al menos ocho semanas) ${ }^{3}$. La cuarta versión revisada del Manual Diagnóstico y Estadístico de Trastornos Mentales (DSM-IV-TR) clasifica al TDM como recurrente ante la reaparición de un episodio depresivo mayor tras al menos dos meses sin cumplir criterio diagnóstico para TDM.

Estudios realizados en el ámbito de la atención primaria reportaron que la TDM es un trastorno altamente recurrente en el que más de la mitad de los pacientes recuperados continúan experimentando uno o más episodios depresivos ${ }^{4,5,6}$. Una adecuada predicción del riesgo de recurrencias podría ser útil para un mejorar el seguimiento de los pacientes con DM, permitiendo clasificarlos en grupos de bajo y alto riesgo. Esto podría limitar el tratamiento farmacológico de los pacientes de bajo riesgo hasta la recuperación del episodio y reservar los tratamientos de mantenimiento para aquellos pacientes con mayor riesgo. También permitiría prevenir recurrencias mediante la reducción de sus factores de riesgo, en aquellos que sea posible. El objetivo de la búsqueda bibliográfica fue documentar la evidencia actual respecto de los predictores que permiten estimar el riesgo de recurrencias en pacientes con antecedentes de TDM.

\section{Resumen de la evidencia}

Hardeveld F, Spijker J, De Graaf R, Nolen WA, Beekman ATF. Prevalence and predictors of recurrence of major depressive disorder in the adult population. Acta Psychiatrica Scandinavica 2010;122:184-191.

Hardeveld y col. identificaron 27 estudios de cohorte que

* Estudiante de la Escuela de Medicina del Instituto Universitario Hospital Italiano de Buenos Aires. nicolas.lescano@hospitalitaliano.org.ar 
habían procurado determinar la incidencia y los predictores de recurrencia del TDM. Éstos debían haber incluido sujetos con TDM diagnosticado mediante entrevistas diagnósticas o cuestionarios basados en los Criterios Diagnósticos de Investigación (RDC) del Colegio de Bedford (BCC), del Manual Diagnóstico y Estadístico de Trastornos Mentales (DSM-III/III$\mathrm{R} / \mathrm{IV}$ ) o de la Clasificación Internacional de Enfermedades (CIE9/10). Su seguimiento debía haber sido igual o superior a seis meses, y por otro lado, debían haber incluido un mínimo de 50 sujetos y haber utilizado los criterios de remisión, recuperación, recaída y recurrencia de Frank et $\mathrm{al}^{3}$.

En los estudios realizados en poblaciones atendidas en unidades de salud mental la incidencia de recurrencias de TDM fue de hasta el $85 \%$ a los 15 años, mientras que en los realizados sobre población general la incidencia de recurrencias a los 15 años fue de $35 \%$.

Los factores más fuertemente asociados con mayor probabilidad de desarrollar recurrencias fueron clínicos, encontrándose dicha asociación en numerosos estudios. En primer lugar, nueve estudios que habían incluido un total de 2073 pacientes con una media de seguimiento de 6,89 años (IC95\% 4,01 a 9,76 años), reportaron una fuerte asociación entre el número de episodios depresivos sufridos previamente y el riesgo de recurrencias, con un OR de hasta 1,64 (IC95\% 1,17 a 2,29). Otro factor fuertemente asociado con un mayor riesgo de recurrencias en seis estudios con seguimientos prolongados (media 5,88 años; IC95\% 2,40 a 9,35) fue la presencia de síntomas subclínicos residuales, con un OR de hasta 3,65 (IC95\% 2,64 a 5,12 ) que se mantuvo luego de ajustar por el potencial efecto confundidor de la cantidad recurrencias previas de episodios depresivos.

Por otro lado, algunas investigaciones documentaron asociaciones más débiles pero estadísticamente significativas: dos estudios que habían incluido un total de 676 pacientes reportaron un mayor riesgo de recurrencia en individuos con deterioro de su funcionamiento en áreas como el trabajo, el ocio y las relaciones sociales (OR 1,12 IC95\% 1,06 a 1,19); dos estudios (552 pacientes) documentaron mayor riesgo de recurrencias en mujeres (OR 1,43 IC95\% 1,10 a $1,86)$ y un estudio (380 pacientes) en sujetos que jamás se hubiesen casado (OR 1,55 IC95\% 1,14 a 2,10). Por otros lado, en una cohorte de 80 pacientes se observó un menor riesgo de recurrencias en pacientes mayores de 45 años de edad (OR 0,11 IC95\% 0,01 a 0,88 ).

El nivel socioeconómico, los eventos de vida adversos y la falta de soporte social no mostraron capacidad predictiva de las recurrencias de TDM en ninguno de los estudios analizados. Dado que los estudios que cumplieron los criterios de inclusión establecidos por los revisores eran difíciles de comparar debido a diferencias entre sus métodos diagnósticos y tiempos de seguimiento, los autores del trabajo consideraron que no era apropiado realizar una síntesis meta-analítica.

Suija K, Alouja A, Kalda R, Maaroos HI. Factors associated with recurrent depression: a prospective study in family practice. Family Practice 2011;28:22-28.
Suija y col. publicaron los resultados de una cohorte prospectiva cuyo objetivo fue determinar los factores asociados a la recurrencia de depresión a los 12 meses del diagnóstico de un TDM. Seleccionaron 1094 pacientes de 18 a 75 años de edad, que habían sido tratados por distintos médicos de familia de Estonia. 142 pacientes (13\%) recibieron el diagnóstico de TDM a través de un cuestionario estructurado (en inglés: Composite International Diagnostic Interview ó CIDI 2.1), basado en el cumplimiento de los criterios diagnósticos del DSM-IV en los últimos seis meses, y fueron seguidos durante 12 meses.

En el $28 \%$ de los pacientes con TDM que ya estaban recuperados a los seis meses del episodio que motivó su reclutamiento en la cohorte se constató un episodio recurrente de depresión durante la evaluación a los 12 meses.

Luego de realizar un análisis multivariado, fueron identificados como predictores independientes de la aparición de recurrencias los antecedentes de abuso de sustancias (OR 7,48 IC95\% 1,42 a 39,43), de haber sido discriminado (OR 2,92 IC95\% 1,05 a 8,11) o abusado durante la infancia (OR 1,58 IC95\% 1,05 a 2,38 ). Al igual que la mayoría de los estudios evaluados en la revisión sistemática anteriormente presentada, no se encontró asociación entre la incidencia de recurrencias y los factores demográficos como el sexo, la edad el nivel socioeconómico.

\section{Comentario}

A partir del análisis de los artículos seleccionados pueden destacarse algunos factores que se asocian con un mayor riesgo de recurrencias tras la recuperación de un episodio depresivo mayor. Por un lado, y como era de esperarse por la selección de una subpoblación con afectación severa, la probabilidad de recurrencia es más del doble ( $85 \%$ a los 15 años) en los pacientes que consultaron a servicios de psiquiatría, respecto de los seleccionados de la población general (35\% a 15 años). A su vez, un mayor número de episodios previos, la presencia de síntomas subclínicos residuales, y el antecedente de abuso de sustancias y/o de haber sufrido discriminación y/o de haber sido abusado durante la infancia se asociaron a una probabilidad aumentada de desarrollar recurrencias al año del diagnóstico de depresión. La mayoría de los predictores mencionados pueden ser perfectamente pesquisados en el ámbito de la atención primaria a través del interrogatorio o bien agregando la inclusión de algún cuestionario específico para depresión que aumente la sensibilidad para la detección de síntomas subclínicos residuales.

Si bien la evidencia encontrada no permite sistematizar un método de cuantificación precisa de la probabilidad que un paciente individual presente una recurrencia, la suma de varios de los predictores mencionados debería hacernos pensar que estamos frente a un paciente con mayor riesgo de desarrollarla. Esta estimación del riesgo de recurrencia permitirá identificar a los pacientes que pueden beneficiarse de conductas terapéuticas destinadas a prevenirlas, por ejemplo tratamientos de mantenimiento (recomendados por revisiones de expertos ${ }^{7}$ ).

3. Frank E, Prien RF, Jarret RB et al. Conceptualization and rationale for consensus definitions of terms in major depressive disorder. Remission, recovery, relapse, and recurrence. Arch Gen Psychiatry 1991; 48: 851-5.

1991; 48: 851-5.

5. Van Weel-Baumgarten E, van den Bosch W, van den Hoogen H, Zitman FG. Ten year follow-up of depression after diagnosis in general practice. Br J Gen Pract 1998; 48 : 1643-6. 6. Wilson I, Duszynski K, Mant AA. 5-year follow-up of general practice patients experiencing depression. Fam Pract 2003; 20:685-9.

7. Blier P, Keller MB, Pollack MH, Thase ME, Zajecka JM, Dunner DL. Preventing Recurrent Depression: Long-Term Treatment for Major Depressive Disorder. Prim Care Companion J Clin Psychiatry 2007; 9(3): 214-223. 